\title{
Association of trait mindfulness with the false recognition of emotional words in bilinguals
}

\author{
Yeh, Li-Hao \\ Chung Yuan Christian University, Taiwan (lhyeh@cycu.edu.tw) \\ $\mathrm{Lu}$, Angela Yi-Chun \\ Chung Yuan Christian University, Taiwan (angelalu@cycu.edu.tw)
}

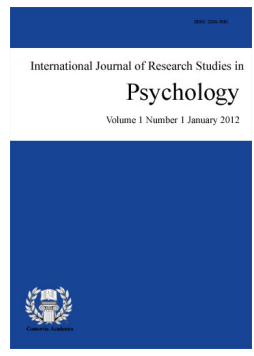

ISSN: $2243-7681$ Online ISSN: 2243-769X

Received: 19 July 2019 DOI: $10.5861 /$ ijrsp. 2019.4009

\section{Abstract}

Mindfulness increases the true memory of emotionally positive stimuli and reduces the negative ones, and enhances false memory. Additionally, few researches have observed a higher false memory of negative critical lures (CLs) compared with positive CLs (i.e., the valence effect). As a consequence, this study examines whether and how trait mindfulness associates with the false memory of emotional words based on their valence values. Chinese-English bilinguals completed the Mindfulness Awareness Attention Scale (MAAS) and participated in a Deese-Roediger-McDermott (DRM) word recognition task in first (L1) as well as second (L2) and cross-language conditions to examine whether the similar effect would be hold in the language that is less emotionally charged. In the Chinese (L1) context, although valence effect did not emerge, trait mindfulness was associated with false recognition of positive, but not with negative CLs. These results suggested that people with high trait mindfulness, through the greater awareness to the positive experience, increased the weight of gist trace in the memory process and, therefore, generate more positive false recognition. Furthermore, this memory bias could be one of the reasons that trait mindfulness associates to less depression and stress and higher well-being. On the contrary, the valence effect emerged, but was not related with trait mindfulness in the English (L2) condition. These unexpected results in the L2 condition are explained by the decoupling of valence and arousal for unbalanced bilinguals, and neutralization of trait mindfulness mainly acted on arousal but not on the valence aspects of emotion.

Keywords: mindfulness; false memory; emotion; bilingualism; DRM 


\section{Association of trait mindfulness with the false recognition of emotional words in bilinguals}

\section{Introduction}

\subsection{Mindfulness and the alteration of emotional information processing}

The concept of mindfulness originates from Buddhism and is a method to cease mental defilements. In modern psychology, mindfulness is often defined as "paying attention in a particular way: on purpose, in the present moment, and non-judgmentally" (Kabat-Zinn, 1990). Mindfulness can be viewed as a mental state attained through practice and a personal disposition in daily life (Brown \& Ryan, 2003). Several mindfulness-related scales have been established to measure the psychological constructs of mindfulness (Baer, Smith, \& Allen, 2004; Baer, Smith, Hopkins, Krietemeyer, \& Toney, 2006; Brown \& Ryan, 2003). Although the constructs among these scales are not identical, two essential components are proposed: (1) self-regulation of attention to observe experiences moment by moment and (2) a non-judgmental and acceptive attitude toward the current experience (Bishop, Lau, Shapiro, Carlson, Anderson, Carmody, \& Devins, 2004).

The literature suggests that trait mindfulness and mindfulness-based training (MBT) are associated with the enhancement of psychological well-being (Brown \& Ryan, 2003; Nyklícek \& Kuijpers, 2008; Shapiro, Carlson, Astin, \& Freedman, 2006). A possible mechanism is that mindfulness may alter the processing of emotional materials. Higher trait mindfulness was observed to be related to fewer negative automatic thoughts (Frewen, Evans, Maraj, Dozois, \& Partridge, 2008), and a psychophysiological study revealed that amygdala reactivity was positively and negatively related to depressive symptoms and trait mindfulness, respectively (Way, Creswell, Eisenberger, \& Lieberman, 2010).

Brief MBT has been related to the deactivation of self-referential default mode network (DMN) regions and the deactivation amygdala, suggesting reduced reactivity and enhanced insula activation that indicates altered interoception (Farb, Anderson, Mayberg, Bean, McKeon, \& Segal, 2010; Farb, Segal, Mayberg, Bean, Mckeon, Fatima, \& Anderson, 2007; Goldin \& Gross, 2010; Goldin, Ramel, \& Gross, 2009; Zeidan, Martucci, Kraft, Gordon, McHaffie, \& Coghill, 2011). For instance, participants with social anxiety disorder (SAD) were presented with positive and negative social trait adjectives and then asked to judge whether they considered these traits to describe them (Goldin et al., 2009). When viewing positive traits, mindfulness-based stress reduction (MBSR) training resulted in reduced activity in DMN regions, indicating less self-referential thinking; however, when viewing negative traits, MBSR training led to increased visual attention, suggesting less avoidance of negative traits. Additionally, Goldin and Gross (2010) observed that participants with SAD that had received MBSR training exhibited reduced amygdala reactivity to negative self-beliefs while focusing on their breath; however, the amygdala reactivity was initially increased then quickly dissipated when they were not focused on breathing. Compared with the baseline, the SAD participants also shifted away from the anterior midline cortical and other DMN regions; this indicated reduced self-referential thinking.

\subsection{Mindfulness and emotional true memory}

In addition to the processing of emotional information, MBT has demonstrated an overall positive effect on long-term memory (Lykins, Baer, \& Gottlob, 2012), autobiographical memory specificity (Heeren, van Broeck, \& Philippot, 2009; Williams, Teasdale, Segal, \& Soulsby, 2000), and episodic memory sensitivity (Brown, Goodman, Ryan, \& Analayo, 2016). Studies have investigated whether a brief mindfulness induction or short-term MBT can modify individuals' memory of emotional materials (Alberts \& Thewissen, 2011; Roberts-Wolfe, Sacchet, Hastings, Roth, \& Britton, 2012). People usually report that emotionally charged 
experiences are easier to recall than neutral events (Choi, Kensinger, \& Rajaram, 2013; Hamann, 2001; Kensinger, \& Corkin, 2003; Kensinger \& Schacter, 2006). Therefore, the rationale shared by Alberts and Thewissen (2011) and Roberts-Wolfe et al. (2012) is that because mindfulness (i.e., either trait or training) can alter individuals' processing of emotional information (e.g., through reduced neural activity toward emotional stimuli), this alteration may indicate downstream effects on the memory of emotional materials.

According to this logic, Alberts and Thewissen (2011) delivered a 12-minute mindfulness induction to nonclinical participants. They subsequently issued a verbal recall task in which positive, negative, and neutral words were presented before the follow-up free recall. In comparison with the participants in the control group, those receiving the mindfulness induction recalled a lower proportion of negative words. Moreover, this effect was limited to negative words, and no difference in the proportion of positive words was found between the control and mindfulness induction groups. According to Alberts and Thewissen (2011), this phenomenon suggests that nonjudgmental attitude cultivated through mindfulness may neutralize the negative valence of a stimulus.

Roberts-Wolfe et al. (2012) conducted a similar study in which nonclinical participants participated in a word recall task, mood and anxiety questionnaire, and psychological well-being scale before and after 12-week MBT. Their results indicated that although the participants in MBT and control groups exhibited no difference on pretests and posttests, the participants in the MBT group recalled a greater number of emotionally positive words in the posttest than the pretest. On the basis of Schroevers and Brandsma (2010), Roberts-Wolfe et al. (2012) suggested that the increased positive and decreased negative memories may be related to the two components of mindfulness, attention regulation and nonjudgmental attitude, respectively. In Schroevers and Brandsma (2010), the participants completed a positive and negative affect schedule, Mindful Attention Awareness Scale (MAAS), two subscales of the Kentucky Inventory of Mindfulness Skills (KIMS), and two subscales of the Self-Compassion Scale before and after an 8-week MBCT. The correlations among the changes in trait mindfulness and the positive and negative affects indicated that increased attention regulation is related to increased positive affect, and a nonjudgmental attitude is related to decrease negative affect.

\subsection{Mindfulness and emotional false memory}

This study extends the research of mindfulness from its effect on emotional true memory to emotional false memory using the DRM paradigm (Roediger \& McDermott, 1995). In the DRM paradigm, the participants first study a series of word lists, followed by a free recall or recognition task that contains non-studied CLs associated with the studied words. False memory occurs when a CL is recalled or recognized during the study. Studies have shown elevated false memory after MBT (Wilson, Mickes, Stolarz-Fantino, Evrard, \& Fantino, 2015; Rosenstreich, 2016).

Mindfulness and false memory in the DRM paradigm - Wilson et al. (2015) used the DRM paradigm to examine whether brief mindfulness induction influences false memory. In the second experiment, participants engaged in either a 15-min breathing meditation or mind-wandering. They found that those who engaged in breathing meditation recalled a greater number of CLs after induction. However, those who engaged in mind-wandering recalled the same number of CLs before and after induction. Wilson et al. (2015) proposed that the nonjudgmental attitude might reduce the memory trace associated with the generation of internal information. Thus, individuals that receive MBT may be more prone to false memories. Rosenstreich (2016) also observed that participants increased false recognition after either a five-week MBT or short mindfulness induction. However, because several studies have found increased semantic fluency after an 8-Week mindfulness intervention (Heeren et al., 2009; Newberg, Wintering, Khalsa, Roggenkamp, \& Waldman, 2010), he suspected that high false memory reflected enhanced cognitive flexibility to activate associated information after MBT.

Additionally, Yeh and $\mathrm{Lu}$ (2017) discovered that a high demand for source monitoring at retrieval alters the correlation between mindfulness and false memory. In their study, the false memory of bilinguals was measured 
through language-specific inquiry (i.e., responding "old" only when a test word was presented in the same language during study); trait mindfulness was revealed to be negatively associated with false memory under within-language conditions and positively associated under cross-language conditions, suggesting that high trait mindfulness may enhance the engagement of verbatim traces to reduce false memory, if even only when the study and test words share the same language.

Although the above studies have demonstrated certain relations between mindfulness and false memory, words used in these studies are not controlled for their emotionality (i.e. valence and arousal aspects). Thus, given that mindfulness is related with the alteration of emotional true memory, it is possible that mindfulness also change the emotional false memory. According to our review of the literature, no studies have investigated whether mindfulness also influences emotional false memory. For that reason, we first review studies related to emotional false memory but not to mindfulness to form our hypotheses about the influence of mindfulness on emotional false memory.

Emotional false memory in the DRM paradigm - Budson, Todman, Chong, Adams, Kensinger, Krangel, and Wright (2006) were the first to utilize the DRM paradigm to investigate whether emotionally charged CLs are more likely to be retrieved by semantically associated words. The results indicated that healthy adults demonstrated greater true recognition of emotional versus neutral CLs but reported no difference between the false recognition of neutral and negative CLs (37\% vs. 39\% for young adults). By contrast, a similar study found a higher false memory of negative CLs than neutral lures (Sharkawy, Groth, Vetter, Beraldi, \& Fast, 2008).

Brainerd, Stein, Silveira, Rohenkohl, and Reyna (2008) argued that the negative and neutral CLs used in Budson et al. (2006) were different in valence and arousal; therefore, they may have limited the ability to determine whether negative CLs are more prone to false memory than are positive and neutral CLs. Therefore, Brainerd et al. (2008) used another set of items in which the mean valence of the CLs and list items was varied, but arousal was similar. They found a valence effect in which participants demonstrated more false recognition for negative CLs, followed by neutral and positive lures. Furthermore, analyses of memory processes have revealed that two main memory processes are responsible for the valence effect: gist similarity and recollection to rejection (i.e., the ability to use verbatim traces to suppress the acceptance of CLs). The weight of gist similarity increases when making recognition decisions for negative CLs, compared with neutral and positive lures. In other words, the gist traces of CLs and their corresponding list items increase in familiarity as the items' valences decrease. However, as the items' valences increase, the recollection rejection gradually increased in weight to the recognition decision. A follow-up study also replicated a similar valence effect (Brainerd, Holliday, Reyna, Yang, \& Toglia, 2010). Although adults demonstrated the valence effect for low-arousal and high-arousal CLs, teenagers demonstrated a valence effect for only high-arousal CLs.

\subsection{The present study}

In this study, based on the aforementioned research, we investigated whether trait mindfulness alters emotional false memory. If the effect of mindfulness on the false memory of valenced words was similar to its effect on the true memory of valenced words (Alberts \& Thewissen, 2011; Roberts-Wolfe et al., 2012), then we would expect to observe that trait mindfulness was either positively associated with the false recognition of positive CLs, negatively associated with the false recognition of negative CLs, or both. Therefore, the valence effect (i.e., the difference between false recognition of positive and negative CLs) would be lower for participants with higher trait mindfulness.

Another purpose of this study was to examine whether the same effect would be true in L2 and cross-language contexts (i.e., difference in language context between study and test).The major difference between L1 and L2 contexts is that bilinguals usually feel less emotionally charged in their L2 (Anooshian \& Hertel, 1994; Aragno \& Schlachet, 1996; Movahedi, 1996; Pavlenko, 2005); therefore, false recognition of valenced CLs in L2 may be less influenced by the emotionality in the gist trace. In this case, the valence effect 
Association of trait mindfulness with the false recognition of emotional words in bilinguals

would be less prominent in the L2 context and not influenced by the participant's level of trait mindfulness. Regarding cross-language conditions, we expected to observe a moderate relationship between trait mindfulness and the false recognition of positive and negative CLs that was less prominent in L1 than in L2 conditions.

\section{Research method}

\subsection{Participants}

A total of 95 students from the Department of Applied Linguistics and Language Studies at the authors' university were recruited. Participants completed a language history questionnaire (LHQ; Li, Zhang, Tsai, \& Puls, 2014). All participants were born and raised in Chinese-speaking communities. To ensure similar language history among participants, those who had lived in an English-speaking environment more than 1 year were excluded. 72 participants' data were used for analyses. Participants' self-reported age of acquisition for speaking, listening and writing for and proficiencies in four language skills are summarized in Table 1. The paired comparison indicates that the participants start to acquire Chinese earlier $(t(71)=-10.7, p<.01)$ and have a higher proficiency in Chinese, measured by a summed rating of four language skills $(t(71)=12.8 .0, p<.01)$.

\section{Table 1}

Participants' language background $(N=72)$

\begin{tabular}{lcc}
\hline \multicolumn{1}{c}{ Language } & Chinese & English \\
\hline Age of Acquisition (year) & & \\
Speaking & 1.8 & 7.5 \\
Reading & 4.5 & 7.7 \\
Writing & 5.5 & 8.0 \\
\hline \multicolumn{1}{c}{ Average Self-Report Proficiency ${ }^{1}$} & & \\
Reading & 6.4 & 5.0 \\
Writing & 6.0 & 4.7 \\
Speaking & 6.6 & 4.6 \\
Listening & 6.8 & 5.0 \\
\hline
\end{tabular}

\subsection{Materials}

Word recognition task - We extracted eight positive and eight negative emotional item lists from the Cornell/Cortland Emotion Lists (Brainerd et al., 2010). The CLs comprised eight high-valence, high-arousal and eight low-valence, high-arousal words. Each item list comprised 15 words. To ensure all the English words were familiar to the participants, 47 other students of English as a foreign language with a similar language background translated all 15 words from each list from English to Chinese. Within each valance category, two lists were excluded from this study phase, because they were used as new words during the test phase. For the remaining 12 lists, only the 10 more frequently correctly translated English words in each list were used in the study phase (the average correct translation rate was $97.3 \%$ ).

The final item lists in the study phase were in English and Chinese and comprised six positive and six negative emotional item lists with 10 words in each list. For valence, the average values for the CLs were 6.2 and 3.2 for positive and negative words, respectively. For arousal, the average values for CLs were 5.0 and 4.6 for the positive and negative words, respectively. The word stimuli in the test phase consisted of 72 words in three types in each language. The list items comprised 36 studied words extracted from the first, fifth, and eighth items in the 12-word list. The CLs comprised 12 CLs semantically associated with each word list. The unlist items comprised 24 unstudied words evenly extracted from four word lists (i.e., two positive and negative word lists, respectively).

Chinese Mindful Attention Awareness Scale - The MAAS (Mindful Attention Awareness Scale) measures 
the natural propensity to be attentive to present moments in daily life (Brown \& Ryan, 2003).It is a unidimensional scale with 15 items. In this study, the Chinese version of the MAAS (CMAAS) was utilized to measure trait mindfulness. The internal consistency of the CMASS is .88 , and 8-week test-retest reliability is .75 (Chang, Lin, \& Huang, 2011).

\subsection{Procedure}

The DRM word recognition task was programmed using E-prime 2.0 software (Psychology Software Tools, Pittsburgh, PA, USA). Each participant was tested individually. They were informed there were 12 lists of 10 words and that half of the lists were in either Chinese or English. The participants were asked to read the lists and remember as many words as possible for the follow-up recognition test.

The words in the study phase were presented orthographically. For each list, participants first viewed "get ready for list A" on a screen, where A corresponds to the list number (1 to 12). Each word appeared for 2 seconds. After viewing the final word list, the participants were presented with 72 test words, one at a time. Approximately $50 \%$ of the participants $(N=35)$ were presented with the English words, and the other half $(N=36)$ were presented with the Chinese words. The participants were asked to press 1 if a test word was presented in the study phase in the same language, press 2 if a test word was presented in the study phase in the other language, and press 3 if the test word was not presented in the study phase. The classification criteria from Kawasaki-Miyaji, Inoue, and Yama (2003) were adopted, in which 1 and 2 are considered "old" responses and 3 is the "new" response.

\section{Result}

Table 2 presents the acceptance rates of the list items, CLs, and new items in all conditions. To reduce baseline differences, we calculated the adjusted acceptance rates for CLs by subtracting the number of recognized new items from that of CLs. In the within-language condition, a 2 (valence: positive and negative) $\times$ 2 (language: Chinese-Chinese and English-English) mixed-design ANOVA was performed. The dependent variable was the adjusted old response to the CLs. This analysis yielded a significant main effect of the valence $\left(F(1,70)=6.5, p<.05\right.$, partial $\left.\eta^{2}=.09\right)$. Participants responded "old" to the negative CLs more often than they did to the positive CLs (.41 vs. $.30,95 \%$ CI for difference $=[.02, .20]$ ). The main effect of the language also reached significance $\left(F(1,70)=13.1, p<.01\right.$, partial $\left.\eta^{2}=.16\right)$, indicating that the participants demonstrated more false recognition of CLs in the Chinese context than they did in the English context (.46 vs. $.25,95 \%$ CI for difference $=[.1, .33])$. Both main effects are consistent with those reported in related studies.

Although the interaction of valence and language only reached marginal significance $(F(1,70)=3.1, p=.08$, partial $\eta^{2}=.04$ ), the descriptive data strongly suggest that the valence effect only occurred in English and not in Chinese. Therefore, pair-wise comparisons were conducted to test our observation. The results indicated that, when conducted in English, the adjusted false recognition of negative CLs was significantly greater than that of positive CLs (.34 vs. .16, $p<.01,95 \%$ CI for difference $=[.06, .31])$. By contrast, when conducted in Chinese, the values for the adjusted false recognition of negative and positive CLs were statistically equivalent (.48 vs. .45). Therefore, the valence effect reported by Brainer et al. (2008; 2010) was not replicated in our Chinese samples, some possible explanations for which are offered in the discussion. The same two-way (valence $x$ language) ANOVA was employed in the cross-language condition. Only the main effect of valence achieved marginal significance $\left(F(1,70)=3.43, p=.068\right.$, partial $\left.\eta^{2}=.05\right)$, indicating a trend that more "old" responses were provided for negative CLs than for positive CLs (.54 vs. .46).

To examine whether trait mindfulness was related to the recognition performance of participants, we first computed the Pearson correlations between CMAAS scores [mean $=59$, standard deviation $(\mathrm{SD})=9.8$ ] and adjusted recognition rates for CLs, and then for list items and valence effects (calculated by subtracting the adjusted recognition rates of positive words from those of negative words) in all study-test-language conditions 
Association of trait mindfulness with the false recognition of emotional words in bilinguals

(Table 3). For false recognition, the results revealed that trait mindfulness was positively correlated with positive CLs $(r=.49, p<.01)$, uncorrelated with negative CLs $(r=.1)$, and was negatively correlated with the valence effect $(r=-.32, p=.05)$ when the test was conducted in Chinese. This indicated that individuals with high trait mindfulness were more likely to generate more false recognition for positive CLs and, therefore, attenuate the valence effect. For true recognition, trait mindfulness was positively associated with list items $(\mathrm{r}=.38, p<.05)$, and no other correlations reached statistical significance.

Table 2

Mean and SD of acceptance rates in study-test language conditions

\begin{tabular}{lccc}
\hline & Items & Chinese-Chinese & English-English \\
\hline Positive & List item & $.80(.22)$ & $.82(.16)$ \\
Negative & & $.82(.20)$ & $.90(.12)$ \\
& Critical lure & $.56(.32)$ & $.30(.31)$ \\
Positive & & $.60(.35)$ & $.50(.32)$ \\
Negative & & English-Chinese & Chinese-English \\
\hline & & & $.66(.19)$ \\
Positive & List item & $.75(.18)$ & $.66(.20)$ \\
Negative & & $.76(.16)$ & $.60(.34)$ \\
Positive & Critical lure & $.58(.39)$ & $.68(.25)$ \\
Negative & & $.68(.34)$ & - English \\
\hline \multicolumn{5}{c}{} & - Chinese & $.14(.15)$ \\
Positive & New item & $.11(13)$ & $.15(.15)$ \\
Negative & & $.12(.17)$ & \\
\hline
\end{tabular}

Table 3

The correlations between CMAAS and adjusted acceptance rates in study-test language conditions

\begin{tabular}{lcccc}
\hline \multicolumn{1}{c}{ Items } & Chinese-Chinese & English-English & Chinese-English & English-Chinese \\
\hline List item & & & & -.06 \\
Positive & $.38^{*}$ & -.08 & .04 & .02 \\
Negative & .25 & .03 & .07 & .08 \\
Valence effect & -.12 & .11 & & -.20 \\
\hline Critical lure & & & -.10 & -.20 \\
Positive & $.49 *$ & .01 & -.10 & .03 \\
Negative & .10 & .11 & .03 & \\
Valence effect & $-.32^{*}$ & .08 & & \\
\hline
\end{tabular}

Note. $* p<.05, * * p<.01$

\section{Discussions}

This study examined whether the asymmetry between the false recognition of positive and negative CLs is influenced by trait mindfulness in within-language and cross-language conditions. The following discussion first addresses the results for the within-language condition followed by the results for the cross-language condition. The false recognition of negative CLs was higher than that of positive CLs, replicating the valence effect reported by Brainer et al. (2008) and Brainer et al. (2010). However, this valence effect was primarily observed in the results for the English condition, and not in those for the Chinese condition. Two possibilities related to the test materials are proposed regarding this result. First, in this study, the list items and CLs were extracted from the study of Brainer et al. (2010) and translated into Chinese; however, these Chinese translations may not have expressed the equivalent emotionality (either valence or arousal) that was present in the original English versions. The recognition of list items seems to provide supportive evidence of this explanation. Although the adjusted acceptance rates for positive and negative Chinese list items were equivalent (.69), the adjusted acceptance rate 
of negative English list items was higher than that of positive ones (.75 vs. .68). Second, Chinese is a logographic language in which words are composed of characters that are often designed to represent meanings; therefore, sometimes words conveying similar emotions or meanings use the same characters. For example, in one list, the list items “狂怒” (rage) and “憤怒” (mad) share the same character “怒,” which, on its own, means anger. Similarly, in another list, the list items “山丘” (hill), “山谷” (valley), “山峰” (peak), and “山羊” (goat) share the character “山," which means mountain. Moreover, in one list, the CL “音樂” (music) and its list items “音符” (note), “聲音” (sound), and “收音機” (radio) all share the same character “音,” which also, on its own, means sound. We suspect that the repetition of characters may have generally influenced false recognition and reduced the salience of the valence effect in the Chinese condition.

The correlational data indicated that trait mindfulness was associated with both true and false recognition of positive words. These findings not only replicate but also extend those published by Roberts-Wolfe et al. (2012) regarding false memory. Additionally, the false recognition of negative CLs was not influenced by trait mindfulness; therefore, the valence effect was reduced as the trait mindfulness of participants increased. As a consequence of these findings, we questioned whether the valence effect would emerge for participants with low trait mindfulness in the Chinese condition. To test this inquiry, participants were divided into two groups at the point of the median score for trait mindfulness, and a two-way (valence and trait mindfulness) repeated ANOVA was conducted with the adjusted false recognition of negative CLs as the dependent variable. A trend of interaction was discerned $(F(1,35)=3.7, p=.06$, partial $\eta 2=.1)$ in that the false recognition of positive CLs was lower than that of negative CLs under conditions of high trait mindfulness (.30 vs. .44). Conversely, for participants with high trait mindfulness, the false recognition of positive CLs was higher than that of negative CLs (.61 vs. .53). One possible mechanism underlying these findings is that attention to present experiences is associated with positive affects (Schroevers \& Brandsma, 2010); therefore, participants with higher trait mindfulness may have a more focused awareness of positive items. Additionally, because low false memory of positive CLs is the result of less weighted gist traces than it is of verbatim traces in the memory process (Brainerd et al., 2008), people with high trait mindfulness, through heightened awareness of the pleasant experience, may assign more weight to gist traces in the memory process and, therefore, generate more false recognitions of positive CLs. The other possibility is that because practicing mindfulness increases participants' semantic fluency (Heeren et al., 2009; Newberg et al., 2010), greater attention to list items leads to a more retrieves of positive list items as well as CLs through the highly active and interconnected semantic network. In summary, our findings also imply that the bias of memory for positive experiences could be one of the reasons why people with high trait mindfulness are less prone to depressive symptoms (Neelarambam, 2015) and stress (Senders, Bourdette, Hanes, Yadav, \& Shinto, 2014), and more prone to enhanced well-being (Brown \& Ryan, 2003).

Furthermore, although we did not discover an association between reduced negative false recognition and trait mindfulness in the current study, it cannot be ruled out as a possibility. As previously mentioned, changes in nonjudgmental attitude were negatively correlated with changes in negative effects (Schroevers \& Brandsma, 2010). Because only CMASS (a measurement that is related to awareness of the present moment) was used to measure trait mindfulness, it is reasonable to assume that positive CLs are only influenced by trait mindfulness. Further studies are required to provide a more in-depth investigation of this topic; for example, multidimensional questionnaires (e.g., the Five Facet Mindfulness Questionnaire; Baer et al., 2006) should be used to examine whether other components (e.g., acting with awareness and not reacting) alter emotional false memory.

Moreover, at the time of writing this manuscript, a study conducted by my colleague and I revealed reduced negative false memory after MBT (Lu, 2017). Aggressive false memory is predictive of both the cyberbullying and traditional bullying of teenagers (Vannucci, Nocentini, Mazzonic, \& Menesini, 2012). Because MBT eases aggressive behavior (Heppner et al., 2008; Yusainy \& Lawrence, 2014), Lu (2017) considered whether MBT can also reduce aggressive false memory. In their study, juvenile delinquents who had committed violent crimes attended 8-week MBT, and their false memory of neutral and aggressive words was measured before and after the training. For all participants (i.e., experimental and control groups), the false memory of aggressive CLs was 
Association of trait mindfulness with the false recognition of emotional words in bilinguals

higher than that of neutral CLs, replicating the valence effect. However, compared with the control group, those who received MBT demonstrated a less notable valence effect in the posttest because of the decreased retrieval of aggressive CLs.

In the English context, higher and lower false recognition of negative and positive CLs echoed the findings of Brainer et al. (2010). However, these results differed from the results for the Chinese context regarding two aspects. First, the valence effect in the English context was more prominent than it was in the Chinese context. Second, the valence effect did not diminish as trait mindfulness increased. The finding that the false recognition of CLs was moderated by valence corresponds with a study on bilingual emotional true memory in which the memory of taboo words and reprimands was more prominent than that of neutral words after an emotional rating task (Ayçiçeği-Dinn \& Caldwell-Harris, 2009). However, the differences in recall between taboo and neutral words and between reprimands and neutral words were more significant in the L1 context than in the L2 context in their study. Therefore, the stronger valence effect in the English context in our study suggests that the effect of emotionality is even stronger in the L2 context than in the L1 context. Nevertheless, this inference is invalid because although our participants had studied English for more than 10 years, they self-rated as being more proficient in Chinese.

If the aforementioned speculative scenario is the case, we must question why the participants exhibited a strong valence effect and why the valence effect was not associated with trait mindfulness in the English (L2) context. The exact reasons for these unexpected findings are unknown, so a potential explanation is proposed. Chinese-English (L1-L2) bilinguals from Hong Kong who rated the valence and arousal of positive, neutral, and negative words presented in both languages demonstrated both a more positive reaction to positive words and a more negative reaction to negative words in English than in Chinese; however, for the arousal rating, the negative words in Chinese were rated as having a greater arousal effect than were the negative words in English (Ong, Hussain, Chow, \& Thompson, 2017). These results suggest that the valence and arousal ratings of bilinguals can be decoupled, at least with regard to negative words. Because the bilingual participants in the this study were living in a similar language environment than were the participants in the study conducted by Ong et al. (2017), they potentially felt less arousal from negative words in English but remained sensitive to valence. In terms of arousal, a psychophysiological study indicated that negative high-arousal pictures elicited higher late positive potential (LPP) responses than did negative low-arousal pictures, and trait mindfulness was associated with decreased LPP responses elicited by negative high-arousal pictures but not by negative low-arousal pictures (Lin, Fisher, Roberts, \& Moser, 2016).

Based on the aforementioned studies, the possible explanation for the findings presented in this study regarding the English condition is that although our bilingual participants may have experienced less arousal on emotional items, they remained sensitive to valence. Moreover, the neutralization of mindfulness primarily affects arousal; therefore, even though a difference was observed in bilingual participants pertaining to the false recognition of positive and negative CLs, their false recognition did not correspond to trait mindfulness. Although this interpretation is intriguing, it fails to provide any direct supporting evidence. Therefore, future research is required to investigate whether mindfulness alters the valence effect when words exhibit low arousal but significant differences in valence. In the cross-language condition, only a marginal difference was observed between the false recognition of positive and negative CLs, suggesting a trend of valence effect caused by the mix of two languages; moreover, similar to the results in English condition, the false recognition of CLs was not associated with trait mindfulness.

Furthermore, we compared the results in the present study with those of another previous study (Yeh \& Lu, 2017); a similarity was observed in that both assessed the relationship between trait mindfulness and DRM false memory for Chinese-English bilinguals. However, the negative correlation of trait mindfulness with false memory under within-language conditions and positive correlation with false memory in cross-language conditions were results that were not replicated in the present study. Instead, a positive correlation between trait mindfulness and false recognition of positive CLs was only observed under the Chinese condition, and not under 
the English or cross-language conditions. One possible reason for this discrepancy is the difference between these two studies regarding source-monitoring requirements at retrieval. In a study conducted by Yeh and Lu (2017), participants were asked to form "old" decisions based on language-specific measurements (i.e., being instructed to only respond "yes" if a word appeared in the same language); therefore, the verbatim trace was essential for establishing a distinction between old and new words. In this study, however, language-specific recognition was encouraged in participants, but if they could not retrieve the vivid verbatim trace then they were permitted to select one option from the two "old" decisions (i.e., respond " 1 " if a test word was presented in the same language in the study or "2" if a test word was presented in a different language in the study). Therefore, the source-monitoring requirement at retrieval may have altered the correlation between trait mindfulness and false memory.

\section{Conclusion: Contribution, limitation and practical advices}

According to our review of relevant literature, this study is the first to demonstrate that the asymmetry in the false recognition of positive and negative CLs is modified by trait mindfulness. The major contribution of this study is to deepen our understanding of the mechanism of emotional regulation of mindfulness. Firstly, we have demonstrated that high trait mindfulness is associated with higher positive, but not negative, false memory. These findings are important because they provide a possible reason why mindfulness practice can be applied to improve emotional regulation. Mindfulness practice may help individuals more easily modify their memory of emotional episodes and with more retrieval of positive episodes, whether real or illusional, they become happier. Secondly, the lack of significant association between emotional false memory and trait mindfulness in the second language (i.e. English) suggests that valence aspect alone is unrelated with trait mindfulness and trait mindfulness selectively associates with the retrievals of positive words only if they are also highly arousal.

In terms of limitation, this study was primarily explorative; although the DRM paradigm and CMASS are both widely used to measure false memory and trait mindfulness respectively in numerous studies, it is not unknown whether the relationships between mindfulness and emotional false memory would be reliably held in other experiment paradigms or measures. Currently, there is no universal definition of mindfulness. In this study, only the present awareness aspect of trait mindfulness was measured. It is possible that other aspects of mindfulness may have different relationships with false memory. Moreover, false memory can be measured by different paradigms, for example, the misleading information paradigm. It is possible that trait mindfulness may not be related with false memory measured by other paradigms. Therefore, more future studies are needed to investigate the relationship between mindfulness and emotional memory.

Although this study is theoretical-orientated, two practical advices are provided, one for mindfulness practice and one for second language learning. Firstly, mindfulness practice has been found to increase one's trait mindfulness (Shahar, Britton, Sbarra, Figueredo, \& Bootzin, 2010). Therefore, even though the effect of mindfulness practice on emotional false memory was not directly examined, our study findings would encourage people to practice mindfulness to retrieve more happy memories, but at the same time, warn them that these enjoyable memories may not be as precise as they believe. Secondly, in addition to learning a L2 in classroom settings, emerging in a natural L2 environment could be important for L2 learners to develop more native-like arousal response toward emotional verbal stimuli.

Acknowledgment - We thank the anonymous reviewers for their valuable feedback on the manuscript. Additionally, we thank the participants and research assistants. This study was approved by the Research Ethics Committee of National Taiwan University (No. 201509ES010). This manuscript was edited by Wallace Academic Editing. 


\section{References}

Alberts, H. J. E. M., \& Thewissen, R. (2011). The effect of a brief mindfulness intervention on memory for positively and negatively valenced stimuli. Mindfulness, 2, 73-77. https://doi.org/10.1007/s12671-011-0044-7

Anooshian, L. J., \& Hertel, P. T. (1994). Emotionality in free recall: Language specificity in bilingual memory. Cognition and Emotion, 8, 503-514. https://doi.org/10.1080/02699939408408956

Aragno, A., \& Schlachet, P. (1996). Accessibility of early experience through the language of origin: A theoretical integration. Psychoanalytic Psychology, 13, 23-34. https://doi.org/10.1037/h0079636

Ayçiçeği, A., \& Harris, C. L. (2004). Bilinguals' recall and recognition of emotion words. Cognition and Emotion, 18, 977-987. https://doi.org/10.1080/02699930341000301

Ayçiçeği-Dinn, A., \& Caldwell-Harris, C. L. (2009). Emotion-memory effects in bilingual speakers: A levels of processing approach. Bilingualism: Language and Cognition, 12, 291-303. https://doi.org/10.1017/S1366728909990125

Baer, R. A., Smith, G. T., \& Allen, K. B. (2004). Assessment of mindfulness by self-report: The Kentucky Inventory of Mindfulness Skills. Assessment, 11, 191-206. https://doi.org/10.1177/1073191104268029

Baer, R. A., Smith, G. T., Hopkins, J., Krietemeyer, J., \& Toney, L. (2006). Using self-report assessment methods to explore facets of mindfulness. Assessment, 13, 27-45. https://doi.org/10.1177/1073191105283504

Bauer, L., Olheiser, E., Altarriba, J., \& Landi, N. (2009). Word type effects in false recall: Concrete, abstract, and emotion word critical lures. The American Journal of Psychology, 122, 469-481. https://doi.org/10.2307/4149007

Bishop, S. R., Lau, M., Shapiro, S., Carlson, L., Anderson, N. D., Carmody. J, \& Devins, G. (2004). Mindfulness: A proposed operational definition. Clinical Psychology: Science, \& Practice, 11, 230-241. https://doi.org/10.1093/clipsy/bph077

Brainerd, C. J., Holliday, R. E., Reyna, V. F., Yang, Y., \& Toglia, M. P. (2010). Developmental reversals in false memory: Effects of emotional valence and arousal. Journal of Experimental Child Psychology, 107, 137-154. https://doi.org/10.1016/j.jecp.2010.04.013

Brainerd, C. J., Stein, L. M., Silveira, R. A., Rohenkohl, G., \& Reyna, V. F. (2008). How does negative emotion cause false memories? Psychological Science, 19, 919-925. https://doi.org/10.1111/j.1467-9280.2008.02177.x

Brown, K. W., \& Ryan, R. M. (2003). The benefits of being present: Mindfulness and its role in psychological well-being. Journal of Personality and Social Psychology, 84, 822-848. https://doi.org/10.1037/0022-3514.84.4.822

Brown, K. W., Goodman, R. J., \& Inzlicht, M. (2013). Dispositional mindfulness and the attenuation of neural responses to emotional stimuli. Social Cognitive and Affective Neuroscience, 8, 93-99. https://doi.org/10.1093/scan/nss004

Brown, K. W., Goodman, R. J., Ryan, R. M, \& Analayo, B. (2016). Mindfulness enhances episodic memory performance: Evidence from a multimethod investigation. PLoS One, 11, 1-19. https://doi.org/10.1371/journal.pone.0160280

Budson, A. E., Todman, R. W., Chong, H., Adams, E. H., Kensinger, E. A., Krangel, T. S., \& Wright, C. I. (2006). False recognition of emotional word lists in aging and Alzheimer disease. Cognitive and Behavioral Neurology, 19, 71-78. https://doi.org/10.1097/01

Cabeza, R., \& Lennartson, E. R. (2005). False memory across languages: Implicit associative response vs fuzzy trace views. Memory, 13, 1-5. https://doi.org/10.1080/09658210344000161

Chang, J., Lin, Y., \& Huang, C. (2011). Zhōngwén băn `zhǐ guān juéchá zhùyì liàng biăo'zhī xìn xiào dù fēnxī [Psychometric properties of the Chinese translation of Mindful Attention Awareness Scale (CMAAS)]. Psychological Testing, 58, 235-260. https://doi.org/10.7108/PT.201104.0090

Choi, H., Kensinger, E. A., \& Rajaram S. (2013) Emotional content enhances true but not false memory for categorized stimuli. Memory \& Cognition, 41, 403-15. https://doi.org/10.3758/s13421-012-0269-2 
Yeh, L.-H., \& Lu, A. Y.-C.

Farb, N. A. S., Anderson, A. K., Mayberg, H., Bean, J., McKeon, D., \& Segal, Z. V. (2010). Minding one's emotions: Mindfulness training alters the neural expression of sadness, Emotion, 10, 25-33. https://doi.org/10.1037/a0019263

Farb, N. A. S., Segal, Z. V., Mayberg, H., Bean, J., Mckeon, D., Fatima, Z., \& Anderson, A., (2007). Attending to the present: mindfulness meditation reveals distinct neural modes of self-reference. Social Cognitive and Affective Neuroscience, 2, 313-322. https://doi.org/10.1093/scan/nsm030

Frewen, P. A., Evans, E. M., Maraj, N., Dozois, D. J. A., \& Partridge, K. (2008). Letting go: Mindfulness and negative automatic thinking. Cognitive Therapy and Research, 32(6), 758-774. https://doi.org/10.1007/s10608-007-9142-1

Goldin, P. R., \& Gross, J. J. (2010). Effects of mindfulness-based stress reduction (MBSR) on emotion regulation in social anxiety disorder. Emotion, 10(1), 83-91. https://doi.org/10.1037/a0018441

Goldin, P. R., Ramel, W., \& Gross, J. (2009). Mindfulness meditation training and self-referential processing in social anxiety disorder: Behavioral and neural effects. Journal of Cognitive Psychotherapy, 23(3), 242-256. https://doi.org/10.1891/0889-8391.23.3.242

Hamann, S. (2001). Cognitive and neural mechanisms of emotional memory. Trends in Cognitive Sciences, 5 , 394-400. https://doi.org/10.1016/S1364-6613(00)01707-1

Hancock, T. W., Hicks, J. L., Marsh, R. L., \& Ritschel, L. (2003). Measuring the activation level of critical lures in the Deese-Roediger-McDermott paradigm. American Journal of Psychology, 116, 1-14. https://doi.org/10.2307/1423332

Harris, C., Gleason, J. \& Ayçiçeği, A. (2006). When is a first language more emotional? Psychophysiological evidence from bilingual speakers. In A. Pavlenko (Ed.), Bilingual minds: Emotional experience, expression, and representation (pp. 257-283). Bristol, UK: Multilingual Matters. https://doi.org/10.21832/9781853598746-012

Heeren, A., van Broeck, N., \& Philippot, P. (2009).The effects of mindfulness on executive processes and autobiographical memory specificity. Behavior Research and Therapy, 47, 403-409. https://doi.org/10.1016/j.brat.2009.01.017

Heppner, W. L., Kernis, M. H., Lakey, C. E., Campbell, W. K., Goldman, B. M., Davis, P., \& Cascio, E. V. (2008). Mindfulness as a means of reducing aggressive behavior: dispositional and situational evidence. Aggressive behavior, 34(5), 486-96. https://doi.org/10.1002/ab.20258

Herndon, F. (2008). Testing mindfulness with perceptual and cognitive factors: External vs. internal encoding, and the cognitive failures questionnaire. Personality and Individual Differences, 44, 32-41. https://doi.org/10.1016/j.paid.2007.07.002

Howe, M. L., Gagnon, N., \& Thouas, L. (2008). Development of false memories in bilingual children and adults. Journal of Memory and Language, 58, 669-681. https://doi.org/10.1016/j.jml.2007.09.001

Kabat-Zinn, J. (1990). Full catastrophe living: Using the wisdom of your body and mind to face stress, pain, and illness. New York, NY: Dell. https://doi.org/10.1002/shi.88

Kawasaki-Miyaji Y., Inoue T., \& Yama, H. (2003). Cross-linguistic false recognition: How do Japanese dominant bilinguals process two languages: Japanese and English? Psychologia, 46, 255-267. https://doi.org/10.2117/psysoc.2003.255

Kensinger, E. A., \& Corkin, S. (2003). Memory enhancement for emotional words: Are emotional words more vividly remembered than neutral words? Memory and Cognition, 31, 1169-1180. https://doi.org/10.3758/BF03195800

Kensinger, E. A., \& Schacter, D. L. (2006). When the Red Sox shocked the Yankees: Comparing negative and positive memories. Psychonomic Bulletin and Review, 13, 757-763. https://doi.org/10.3758/BF03193993

Kroll, J. F., \& Stewart, E. (1994) Category interference in translation and picture naming: evidence for asymmetric connections between bilingual memory representations. Journal of Memory and Language, 33, 149-174. https://doi.org/10.1006/jmla.1994.1008

Kroll, J. F., \& Tokowicz, N. (2001). The development of conceptual representation for words in a second language. In J. L. Nicol (Ed.), One mind, two languages: Bilingual language processing (pp. 49-71). 
Association of trait mindfulness with the false recognition of emotional words in bilinguals

Cambridge, MA: Blackwell. https://doi.org/10.1353/lan.2005.0034

Li, P., Zhang, F., Tsai, E., \& Puls, B. (2014). Language history questionnaire (LHQ 2.0): A new dynamic web-based research tool. Bilingualism: Language and Cognition, 17(3), 673-680.

https://doi.org/10.1017/S1366728913000606

Lin, Y., Fisher, M. E., Roberts, S. M. M., \& Moser, J. S. (2016). Deconstructing the Emotion Regulatory Properties of Mindfulness: An Electrophysiological Investigation. Frontiers in Human Neuroscience, 10: 451. https://doi.org/10.3389/fnhum.2016.00451

Lu, C. (2017). The influence of mindfulness practice on anger rumination, false memory and aggression. (Unpublished master thesis). Chung Yuan Christian University, Taoyuan, Taiwan. https://doi.org/10.1007/s12671-016-0629-2

Lykins, E. L., Baer, R. A., \& Gottlob, L. R. (2012). Performance-based tests of attention and memory in long-term mindfulness meditators and demographically matched non-meditators. Cognitive Therapy and Research, 36, 103-114. https://doi.org/10.1007/s10608-010-9318-y

Mao, W. B., Wang, S., \& Kang, L. L. (2012). Effect of item-specific processing and relational processing on cross-language false memory among less proficient Chinese-English bilinguals. Acta Psychologica Sinica, 44, 1289-1296. https://doi.org/10.3724/SP.J.1041.2012.01289

Marian, V., \& Kaushanskaya, M. (2004). Self-construal andemotion in bicultural bilinguals. Journal of Memory and Language, 51, 190-201. https://doi.org/10.1016/j.jml.2004.04.003

Marmolejo, G., Diliberto-Macaluso, K., \& Altarriba, J. (2009). False memory in bilinguals: Does switching languages increase false memories? The American Journal of Psychology, 122, 1-16. https://doi.org/10.1080/09658211.2011.653373

Movahedi, S. (1996). Metalinguistic analysis of therapeutic discourse: Flight into a second language when the analyst and the analysand are multilingual. Journal of the American Psychoanalytic Association, 44, 837-862. https://doi.org/10.1177/000306519604400308

Neelarambam, K. (2015). Trait mindfulness as a mediator of resilience, depressive symptoms, and trauma symptoms (Unpublished doctoral dissertation). Georgia State University, Atlanta, GA. https://doi.org/10.1007/s12671-012-0153-y

Newberg, A. B., Wintering, N., Khalsa, D. S., Roggenkamp, H., \& Waldman, M. R. (2010). Meditation effects on cognitive function and cerebral blood flow in subjects with memory loss: a preliminary study. Journal of Alzheimer's Disease, 20, 517-526. https://doi.org/10.3233/JAD-2010-1391

Nyklícek, I., \& Kuijpers, K. F. (2008). Effects of mindfulness-based stress reduction intervention on psychological well-being and quality of life: Is increased mindfulness indeed the mechanism? Annals of Behavioral Medicine, 35, 331-340. https://doi.org/10.1007/s12160-008-9030-2

Ong, E., Hussain, S., Chow, Y., \& Thompson, C. (2017, March). Variations in bilingual processing of positive and negative information. Paper presented at 6th Annual International Conference on Cognitive and Behavioral Psychology, Singapore. https://doi.org/10.5176/2251-1865_CBP17.14

Pavlenko, A. (2005). Emotions and multilingualism. Cambridge, MA: Cambridge University Press. https://doi.org/10.1017/CBO9780511584305

Psychology Software Tools, Inc. [E-Prime 2.0]. Retrieved from http://www.pstnet.com

Reyna, V. F., \& Brainerd, C. J. (1995). Fuzzy-trace theory: An interim synthesis. Learning and Individual Differences, 7, 1-75. https://doi.org/10.1016/1041-6080(95)90031-4

Roberts-Wolfe, D., Sacchet, M., Hastings, E., Roth, H., \& Britton, W. (2012). Mindfulness training alters emotional memory recall compared to active controls: Support for an emotional information processing model of mindfulness. Frontiers in Human Neuroscience, 6:15. https://doi.org/10.3389/fnhum.2012.00015

Roediger, H. L., \& McDermott, K. B. (1995). Creating false memories. Journal of Experimental Psychology: Learning, Memory, \& Cognition, 22, 814-816. https://doi.org/10.1037/0278-7393.22.3.814

Rosenstreich, E. (2016). Mindfulness and false-memories: The impact of mindfulness practice on the DRM paradigm. The Journal of Psychology, 150, 58-71. https://doi.org/10.1080/00223980.2015.1004298

Sahlin, B. H., Harding, M. G., \& Seamon, J. G. (2005). When do false memories cross language boundaries in 
Yeh, L.-H., \& Lu, A. Y.-C.

English-Spanish bilinguals? Memory \& Cognition, 33, 1414-1421. https://doi.org/10.3758/BF03193374

Schroevers, M. J., \& Brandsma, R. (2010). Is learning mindfulness associated with improved affect after mindfulness-based cognitive therapy? British Journal of Psychology, 101, 95-107. https://doi.org/10.1348/000712609X424195

Senders, A., Bourdette, D., Hanes, D., Yadav, V., \& Shinto, L. (2014). Perceived stress in multiple sclerosis: The potential role of mindfulness in health and well-being. Journal of Evidence-Based Complementary \& Alternative Medicine, 19, 104-111. https://doi.org/10.1177/2156587214523291

Shahar, B., Britton, W. B., Sbarra, D. A., Figueredo, A. J., \& Bootzin, R. R. (2010). Mechanisms of change in mindfulness-based cognitive therapy for depression: Preliminary evidence from a randomized controlled trial. International Journal of Cognitive Therapy, 3(4), 402-418. https://doi.org/10.1521/ijct.2010.3.4.402

Shapiro, S. L., Carlson, L. E., Astin, J. A., \& Freedman, B. (2006). Mechanisms of mindfulness. Journal of Clinical Psychology, 62, 373-386. https://doi.org/10.1002/jclp.20237

Sharkawy, J. E., Groth, K., Vetter, C., Beraldi, A., \& Fast, K. (2008). False memories of emotional and neutral words. Behavioural Neurology, 19, 7-11. https://doi.org/10.1155/2008/587239

Vannucci, M., Nocentini, A., Mazzoni, G., \& Menesini, E. (2012). Recalling unpresented hostile words: False memories predictors of traditional and cyberbullying. European Journal of Developmental Psychology, 9, 182-194. https://doi.org/10.1080/17405629.2011.646459

Way, B. M., Creswell, D., Eisenberger, N. I., \& Lieberman, M. D. (2010). Dispositional mindfulness and depressive symptomatology: Correlations with limbic and self-referential neural activity during rest. Emotion, 10, 12-24. https://doi.org/10.1037/a0018312

Williams, J. M. G., Teasdale, J. D., Segal, Z. V., \& Soulsby, J. (2000). Mindfulness-based cognitive therapy reduces over general autobiographical memory in formerly depressed patients. Journal of Abnormal Psychology, 109, 150-155. https://doi.org/10.1037/0021-843X.109.1.150

Wilson, B. M., Mickes, L., Stolarz-Fantino, S., Evrard, M., \& Fantino, E. (2015). Increased false-memory susceptibility after mindfulness mediation. Psychological Science, 26, 1567-1573. https://doi.org/10.1177/0956797615593705

Yeh, L., \& Lu, A. Y. (2017). Relationship between trait mindfulness and false memory: A bilingual Deese-Roediger-McDermott paradigm. Universal Journal of Psychology, 5, 105-113. https://doi.org/10.13189/ujp.2017.050302

Yusainy, C., \& Lawrence, C. (2014). Relating mindfulness and self-control to harm to the self and to others. Personality and Individual Difference, 64, 78-83. https://doi.org/10.1016/j.paid.2014.02.015

Zeidan, F., Martucci, K. T., Kraft, R. A., Gordon, N. S., McHaffie, J. G., \& Coghill, R. C. (2011). Brain mechanisms supporting the modulation of pain by mindfulness meditation. The Journal of Neuroscience, 31, 5540-5548. https://doi.org/10.1523/JNEUROSCI.5791-10.2011 